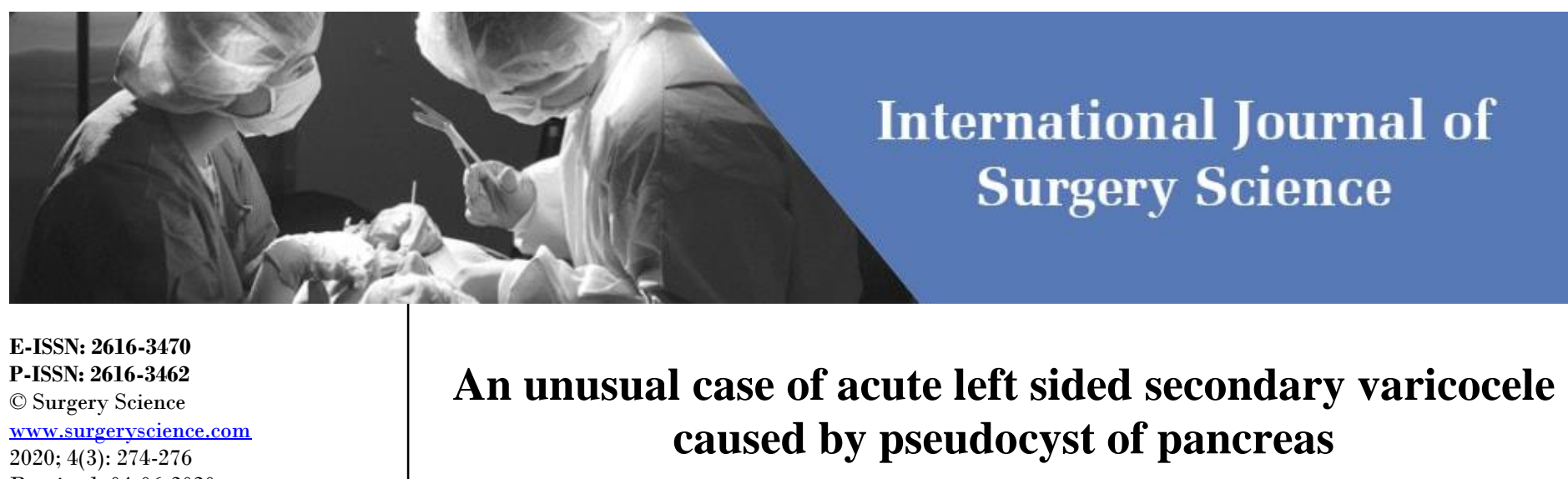

Received: 04-06-2020

Accepted: 24-07-2020

BJ Sharath Chandra

Professor, Department of General Surgery, JSS Academy of Higher

Education and Research, JSS

Medical College Mysuru,

Karnataka, India

Pankaja SS

Assistant Professor, Department of General Surgery, JSS Academy of Higher Education and Research, JSS Medical College Mysuru,

Karnataka, India

\section{Sajan Sehgal}

Former Junior Resident,

Department of General Surgery, JSS Academy of Higher Education and Research, JSS Medical College

Mysuru, Karnataka, India

Thulasi Vasudevaiah Associate Professor, Department of General Surgery, JSS Academy of Higher Education and Research. JSS Medical College Mysuru, Karnataka, India

\section{Corresponding Author:} Sajan Sehgal

Former Junior Resident, Department of General Surgery, JSS Academy of Higher Education and Research, JSS Medical College Mysuru, Karnataka, India

\section{BJ Sharath Chandra, Pankaja SS, Sajan Sehgal and Thulasi Vasudevaiah}

\author{
DOI: https://doi.org/10.33545/surgery.2020.v4.i3e.506
}

\section{Abstract}

Pancreatic pseudocysts are common complications of both acute and chonic pancreatitis. They frequently get complicated when large in size and persistent beyond 6 weeks. Varicoceles are chronic conditions, occurring more frequently on the left side due to obvious anatomical reasons. Rarely varicoceles occur secondary to renal and retroperitoneal masses. Here we are reporting an unusual case of left sided acute varicocele occurring in a young adult, an alcoholic, secondary to pancreatic pseudocyst. Varicocele resolved completely in the immediate postoperative period following laparoscopic cysto-gastrostomy.

Keywords: Cysto-gastrostomy, complicated Pseudocyst, acute Varicocele, secondary varicocele, pancreatic pseudocyst

\section{Introduction}

Case Summary: A 27 year old adult male was admitted to our surgical unit with complaints of upper abdominal pain, nausea and vomiting for 6 weeks. He was a chronic alcoholic for 5 years. He was diagnosed and treated for acute ethanol induced pancreatitis 6 weeks earlier. However mild pain persisted and increased in intensity gradually. Vomiting was present only for solids and tolerated liquids well. Vomitus contained fresh food particles and was non-bilious and not blood tinged. There was no associated fever. He also noticed swelling of the left side of the scrotum since 15 days. Sudden in onset and nonprogressive in nature. There was no associated pain. There was no history of preceding trauma.

\section{Clinically, patient was icteric mildly}

On inspection of the abdomen there was fullness in the epigastric region and there was no visible gastric peristalsis. On palpation there was no tenderness or guarding; a large mass was palpable in the epigastric region approximately $10 \mathrm{X} 8 \mathrm{~cm}$. It was dull to percuss. There was no free fluid and no palpable hepato-splenomegaly.

On examination of the scrotum with patient in standing posture there was evidence of left sided grade III varicocele with a typical 'bag of worms' feeling on palpation [Fig:1]. The varicocele was present even after the patient was made to lie down. Both testes were normal. Relevant blood investigations were done; liver function tests (LFT) showed total serum bilirubin of 2.2 $\mathrm{mg} \%$, direct bilirubin of $1.02 \mathrm{mg} \%$. Serum amylase and serum lipase levels were normal.

Abdominal ultrasound revealed atrophic body and tail with normal head of pancreas; well defined thick walled $(7 \mathrm{~mm})$ cyst in the epigastric region measuring $17 X 9 X 13 \mathrm{~cm}$ (volume 1200CC). Spleen enlarged, 14.6 x $5.8 \mathrm{~cm}$. The colour doppler test confirmed the presence of left sided varicocele. Contrast enhanced abdomino-pelvic computed tomography (CT scan) revealed a huge pseudocyst, measuring $17 \times 11 \times 14 \mathrm{~cm}$, along the whole body of pancreas with regional peripancreatic inflammatory changes. It was seen compressing the pancreatic parenchyma and the stomach, displacing the superior mesenteric artery posteriorly, indenting the transverse colon inferiorly, with thin internal septations and soft tissue component, occupying the left subdiaphragmatic space. Splenic artery was normaland splenic vein compressed. However there was no evidence of thrombosis. No foci of intraductal or intraparenchymal calcification. In an attempt to explore the cause for varicocele, CT scan was reviewed. There was indentation of the left renal vein before the confluence to inferior vena cava without thrombosis, with retrograde dilatation of both renal and left testicular veins causing varicocele [Fig:2]. 
Perigastric, mesenteric and retroperitoneal collaterals noted. Upper GI endoscopy revealed early esophagealvarices, fundal varices, mild portal gastropathy and extrinsic compression of the body of the stomach and first part of the duodenum. Initially endoscopic drainage was planned and attempted. However due to technical reasons, it was deferred and surgery planned. Due to financial reasons, patient got discharged at request and readmitted after a week. Repeat ultrasound scan showed increase in the size of the cyst and volume to 2300cc. Following preoperative preparations and adequate counselling, the patient was subjected for laparoscopic cysto-gastrostomy. Intraoperatively a large cyst was noticed in the lesser sac area with compression and anterior displacement of stomach. Anterior gastrotomy done. Over the bulging posterior gastric wall, incision made and deepened through the cyst wall. 2 liters of fluid sucked out. Cystogastrostomy created between cyst wall and the thickened posterior wall of the stomach [Fig:3]. Anterior gastrotomy site closed, drain placed in the subhepatic region and laparotomy wound closed. On the first Post-operative day, examination of the scrotum revealed complete resolution of the left varicocele, indicating that the mechanical pressure of the cyst on the left renal vein was relieved by the surgery. Patient had a satisfactory postoperative period and was discharged after a week.

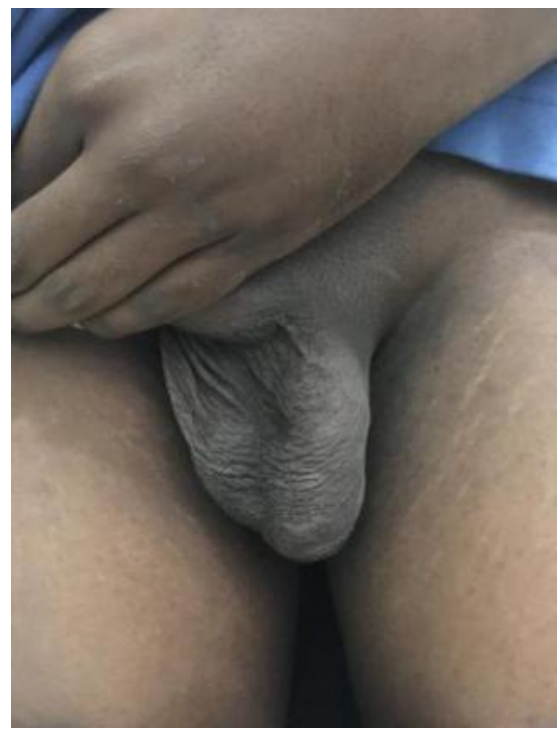

Fig 1: (L)Varicocele.

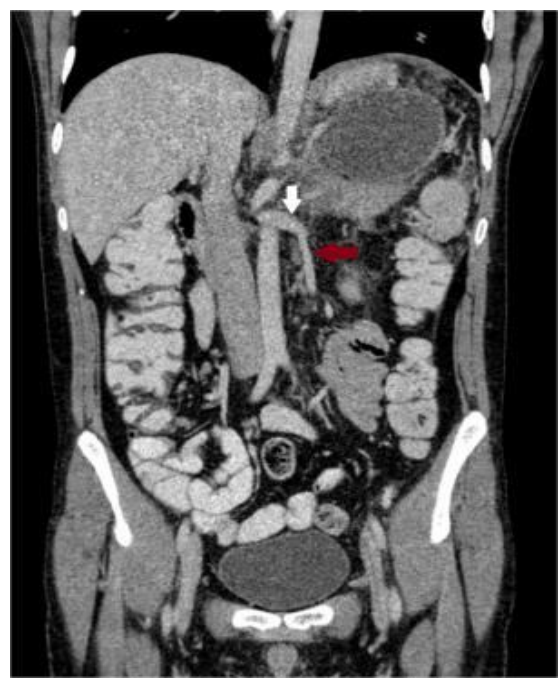

Fig 2: CECT abdomen with Dilated (L)testicular vein(arrow)

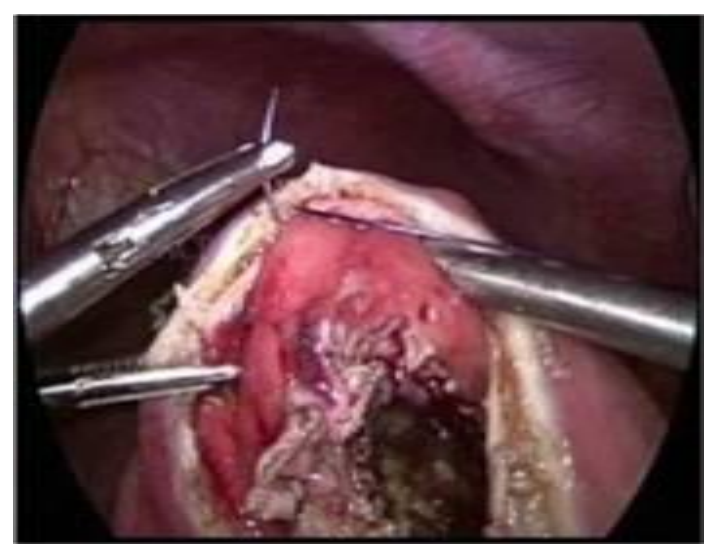

Fig 3: Lap. Cysto-gastrostomy

\section{Discussion}

Pancreatic pseudocyst is a collection of pancreatic inflammatory fluid and necrotic debris, usually encapsulated within the lesser sac or retroperitoneal region. It occurs in $6-8 \%$ of patients following acute pancreatitis and $20-40 \%$ of chronic pancreatitis. Acute pseudocysts, if they are small, will resolve over a period of 6 weeks. Larger cysts $(>10 \mathrm{~cm})$ are unlikely to resolve and require intervention by endoscopic, laparoscopic or open approach.

Primary varicocele is a common scrotal condition, affecting about $15 \%$ of young adult males. It represents dilatation and tortuosity of the pampiniform plexus of the spermatic vein. The condition is much more common on the left $(90 \%)$, because left testicular vein joins the left renal vein at right angle and 8 to 10 $\mathrm{cm}$ higher up compared to the right testicular vein which joins the inferior vena cava obliquely at a lower level ${ }^{[1]}$. Varicocele is usually asymptomatic and may present with mild pain. It is often discovered during clinical evaluation for infertility. In adults, surgical treatment of varicocele is indicated only for relief of pain or treatment of low sperm count ${ }^{[2]}$.

Rarely, varicoceles may be caused secondarily by an invading renal cell carcinoma, retroperitoneal tumors, pressure by the lymph nodal mass or a hydronephrotic kidney ${ }^{[1,3]}$.

Hence it is a common practice to rule out renal and retroperitoneal masses by doing an ultrasound scan, especially in older individuals ${ }^{[4]}$.

Pseudocyst can compress on the adjacent structures like stomach, duodenum, bile duct, portal vein and colon, and present with obstructive features. Pseudocyst of pancreas causing varicocele is very rare and unusual. There have been only 2 case reports of pancreatic pseudocyst causing left sided acute varicocele, secondary to compression of left renal and testicular veins. First case was reported in the year 1983, where a young alcoholic developed repeated episodes of pancreatitis secondary to pancreas divisum, which got complicated with pseudocyst formation and left varicocele ${ }^{[5]}$. Second case was reported in the year 2013, where a ypung aloholic male patient presented with heaviness in the left side of scrotum and on imaging the abdomen they found the presence of chronic pancreatitis with dilated MPD and a pseudocyst compressing the left renal and testicular veins resulting in varicocele. In both the cases, varicocele resolved following surgical decompression of the pancreatic pseudocyst ${ }^{[6]}$. There are reports on other rare causes of secondary varicocele. A right sided varicocele caused by renal cell carcinoma with tumor thrombus only in the right spermatic vein without extension in inferior vena cava, an unusual finding, has been reported ${ }^{[7]}$. very rarely, in children too, varicoceles can occur with renal masses like Wilm's tumor 
[8]. Unusually Pancreatic head malignancy can cause unilateral right sided varicocele ${ }^{[9]}$. A 45 year old male had a left sided varicocele due to compression on left renal vein by a large cystic lesion in the left lobe of liver which turned out to be biliary cystadenoma ${ }^{[10]}$.

These reports support the rule of examining the abdomen both clinically and by ultrasound in all cases of varicoceles to look for masses causing compression or direct invasion and occlusion of either or both renal and testicular veins resulting in varicocele.

\section{Conclusion}

In conclusion, reports on acute varicoceles are rare and pancreatic pseudocyst causing secondary varicocele by compressing the terminal part of left renal vein is still rare and unusual as observed in our case and in literature. In any case of varicocele, doing thorough abdominal examination and ultrasound scan, in all age groups helps to differentiate the primary and secondary varicoceles and if found, treating the abdominal pathology will decompress the secondary varicocele spontaneously.

Patient's Consent: Informed consent was obtained from the patient to publish the Clinical images.

Conflict of interest: None.

Institutional funding: None.

\section{References}

1. Aza Mohammed, Frank Chinegwundoh. Testicular Varicocele: An Overview. Urol Int. 2009; 82:373-379

2. Vakalopoulos I, Kampantais S, Lymperi S et al. Should we expand the indications for varicocele treatment?. Transl Androl Urol. 2017; 6(5):931-942.

3. Pauroso S, Di Leo N, Fulle I, Di Segni M, Alessi S, Maggini E. Varicocele: Ultrasonographic assessment in daily clinical practice. J Ultrasound. 2011; 14(4):199-204.

4. El-Saeity NS, Sidhu PS. Scrotal Varicocele, Exclude a Renal Tumour. Is This Evidence Based?. Clin Radiol. 2006; 61(7):593-9.

5. Dixon JM, Armstrong CP. 0 Eremin. Case report: Varicocoele caused by a pancreatic pseudocyst. Gut. 1983; 24:438-440.

6. Yashant Aswani, Priya Hira et al. Secondary varicocele caused by Pancreatic Pseudocyst obstructing testicular venous drainage. Journal of the pancreas. 2013; 14(6):674675.

7. Hideo Shisaka, Naohiro fujimoto et al. A rare case of right varicocele testis caused by a renal cell carcinoma thrombus in the spermatic vein-case report. International journal of urology 2006; 13:844-845.

8. Vijai Williams et al. PGIMER, Varicocele with an abdominal mass; The Journal of Paediatrics. 2018; 198:317.

9. Kelly E Davis, Charles T Simpkin et al. Unilateral right sided varicocele associated with pancreatic cancer- A cadaveric case report. Translational Research in anatomy. 2017; 7:1-4.

10. Nanvati AJ, Sanjay Nagral. Varicocele due to biliary Cystadenoma. BMJ Case Rep. 2014; 20(PMID-2541422)internet 